\title{
The Cosmochemical Record of Carbonaceous Meteorites: An Evolutionary Story
}

\author{
Sandra Pizzarello \\ Department of Chemistry \& Biochemistry, Arizona State University, Tempe, AZ 85287-1604. USA. pizzar@asu.edu.
}

Received October 24, 2009; accepted December 18, 2009

\begin{abstract}
This account traces a lecture given to El Colegio Nacional last March during a Conference "On the origin of life on the Earth" organized to celebrate Darwin's Bicentennial. It reports on the extraterrestrial organic materials found in carbon-containing meteorites, their composition, likely origin and possible prebiotic contribution to early terrestrial environments. Overall, this abiotic chemistry displays structures as diverse as kerogen-like macromolecules and simpler soluble compounds, such as amino acids, amines and polyols, and show an isotopic composition that verifies their extraterrestrial origin and lineage to cosmochemical synthetic regimes. Some meteoritic compounds have identical counterpart in the biosphere and encourage the proposal that their exogenous delivery to the early Earth might have fostered molecular evolution. Particularly suggestive in this regard are the unique L-asymmetry of a number of amino acids in some meteorites as well as the rich and almost exclusively water-soluble compositions discovered for other meteorite types.
\end{abstract}

Key words: Cosmochemical, Life, Earth, Abiotic Chemistry, Macromolecules.

\section{Introduction}

Much has been said in Darwin's honour in the past year as we came, once again, to recognize his towering scientific accomplishments. Less noted amidst the celebrations was the fact that Darwin, in one of his intuitions for fundamental questions, also addressed the theme of the origin of life. He touched upon it only once, in a private occasion writing to a friend [1], but undeniably foretold the idea of extending biological evolution to prebiotic chemistry. It is disappointing and frustrating that, in spite of the tremendous advances in evolutionary biology that followed "On origin of species", the studies on the origin of life are still fraught to this date with equally large unknowns and general uncertainties, if not larger, as the ones Darwin faced in his efforts and we are still exploring his idea today.

The actual origin of life, if we mean to define it as the emergence of a self-reproducing complex chemistry that is capable of evolving and sustaining life's functions, is still utterly unknown. It will not be easy to decipher either, because there are inherent difficulties in the facts that emergent life processes may have differed in their core molecular organization and function from even the simplest of extant life forms, from which they are so far removed, and that the records of early Earth primitive chemistry have been destroyed by over four billion years of ensuing geological and biological changes.
Resumen. Esta reseña resume una conferencia impartida en marzo pasado en El Colegio Nacional en el Simposio "Sobre el origen de la vida en la Tierra" organizado para la celebración del Bicentenario del nacimiento de Darwin. Se informa sobre los materiales orgánicos extraterrestres encontrados en meteoritos que contienen carbono, su composición, origen probable y su posible contribución prebiótica al ambiente terrestre incial. Esta química abiótica incluye estructuras diversas, tales como macromoléculas querogénicas y compuestos solubles simples, como amino ácidos, aminas y polioles, y muestran una composición isotópica que confirma su origen extraterrestre y su linaje sintético cosmoquímico. Algunos compuestos meteoríticos tienen contrapartes idénticas en la biósfera y apoyan la propuesta referente a que su envío exógeno a la Tierra primitiva podría haber favorecido la evolución molecular. Particularmente sugerente en este aspecto es la asimetría L exclusiva como su rica y casi exclusiva composición soluble en agua también descubierta en otros tipos de meteoritos.

Palabras clave: Cosmoquímica, vida, Tierra, química abiótica, macromoléculas.

The quest for life's origin, therefore, has to rely only on hypotheses and these must reasonably be drawn from what we as yet know of the chemical processes that surrounded that beginning: the preceding chemical evolution and the biological evolution that followed. Darwin's assumption is in line with our current approach, which is based on the record of life's fundamental evolutionary nature throughout its cellular history and the reasonable assumption that the same property extended to its beginnings. We have also learned much about extraterrestrial chemistry in the last decades, we know now that life's elements $\mathrm{C}, \mathrm{H}, \mathrm{N}, \mathrm{O}$, and $\mathrm{S}$, have a long cosmic history during which complex organic molecules are formed in diverse environments, from the gas and dust of the interstellar clouds to small solar system bodies [2], and this understanding of an active cosmochemical evolution has further led to question whether those two evolutionary processes surrounding life's origin ever actually met and, if so, when and how. It is in this exobiological context that the study of carbonaceous meteorites has offered a somewhat surprising study guide for both setting the prebiotic stage of the early Earth and answering at least some of our questions by providing to direct analysis a natural sample of chemical evolution.

Most meteorites are fragments of asteroids, planetesimals of odd size and shape that orbit the Sun in great number between Mars and Jupiter and are the remnants of a planet that never formed. It appears that, during the formation of the solar system, the aggregating "embrios" [3] of nebular materials on 
the way to form a planet in this region fell under the gravity of the giant planets and were either scattered in various directions or left unable to coalesce. This dynamical instability brought in objects from outer orbits as well, such as the transNeptunian asteroids [4] that have been seen to periodically behave as comets [5], also leading to continual collisions and the delivery of their fragments to the Earth through the ages. Although some of the asteroids did grow big enough to form an iron core (from them come the familiar iron meteorites), most never had their composition drastically transformed by gravitational high temperatures or pressures and their meteoritic fragments are expected to contain unaltered a pristine record of early Solar System chemistry. This is particularly true for the carbonaceous chondrites (CC), a primitive sub-group of stony meteorites of elemental composition very similar to the one of the Universe overall that have the distinction of containing several percent amounts $(\sim 1.5-4 \%)$ of carbon, the most part of which is present as organic materials. Uniquely, therefore, the studies of these meteorites have entered the discussion on the origin of life, as they tell us of the organic chemistry that came to be in the Solar System, at the time the Earth was formed and before the inception of life.

$\mathrm{CC}$ organic carbon content was recognized since their first analyses in the nineteenth century [6], however, the unequivocal discovery of abiotic extraterrestrial organic material came with the fall of the Murchison meteorite, in September 1969 [7]. This was a propitious fall because, at the time, many laboratories in the US were preparing for returning lunar samples and the possibility that they could contain organic compounds; so it happened that NASA scientist analyzed directly a pristine Murchison sample, thinking of using it as a likely analog of Moon material. No one would connect lunar soils with the prospect of organic syntheses any longer; however, this meteorite was collected in the amount of $100 \mathrm{~kg}$ and has been comprehensively studied now for forty years, allowing a significant understanding of extraterrestrial organic chemistry and the formative history of CC in particular [8]. Recently, surprisingly novel data were obtained from stones recovered in Antarctica [9-10] that belong to a different CC family: the CR or Renazzo-type meteorites. In the brief review that follows, the organic composition of the Murchison meteorite and Antarctic finds will be described separately, not only because the latter are as yet partially characterized but also to convey the current understanding of the abiotic organic chemistry of $\mathrm{CC}$, which appears to be heterogeneous in composition, distribution and, possibly, formative history.

\section{The organic composition of the Murchison meteorite.}

The larger fraction of Murchison organic carbon, about $70 \%$ of total, is present as a complex and insoluble macromolecular material that is obtained from meteorite powders only after repeat extraction with solvents and demineralization with acids. It is often referred to as kerogen-like because, in addition to $\mathrm{C}$, it also contains $\mathrm{H}, \mathrm{O}, \mathrm{N}$, and $\mathrm{S}$. Being insoluble, it can be characterized only in toto by infrared and nuclear magnetic resonance spectroscopy, pyrolytic decomposition or microscopy. By this combination of techniques, it appears as a fluffy undifferentiated material (Fig.1a) [11] and to have the approximate composition shown in Figure 1b [12], i.e., to consist of condensed aromatic, hydroaromatic, and heteroaromatic macromolecules containing alkyl branching, functional groups, such as $\mathrm{OH}$ and $\mathrm{COOH}$, that are bridged by alkyl chains and ether or sulfur linkages. Macromolecular carbonaceous material free of minerals can also be found in untreated Murchison powders as distinct and compositionally heterogeneous nanosized entities such as aromatic flakes, solid and hollow spheres or tubes [11] that, by visual estimate, appear to have an abundance of up to $10 \%$ of total insoluble carbon (Fig.1a). Other minor components of this insoluble material are sometimes described as exotic carbon species for their relation to isotopically exotic $\mathrm{He}, \mathrm{Xe}$, and $\mathrm{Ne}$ : the nanodiamonds, silicon carbide, graphite and fullerenes, which are considered of presolar origin and may have formed in the outflow of carbon stars [8].

Overall, these compositional characteristics would seem to imply that $\mathrm{CC}$ insoluble carbon was unlikely to contribute to

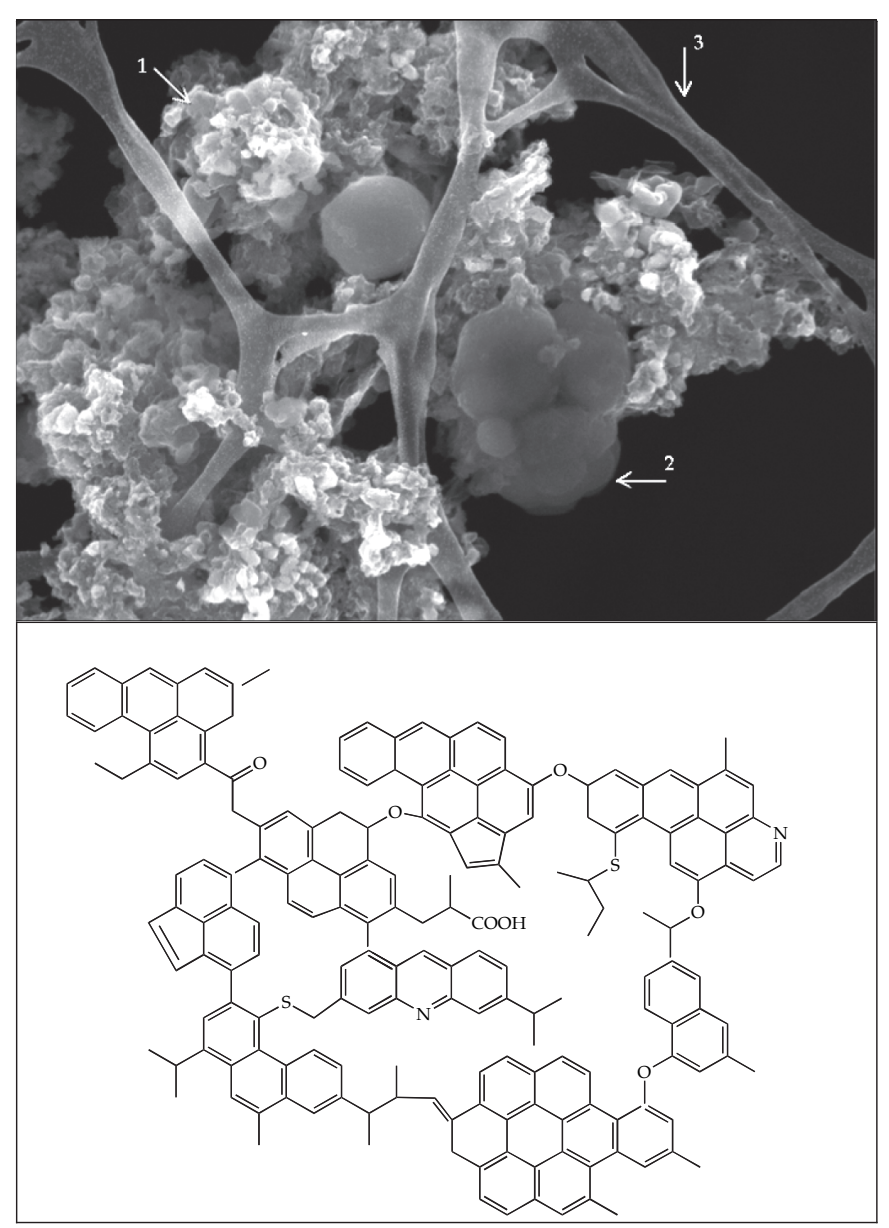

Fig. 1. a) Scanning electron microscope image of Murchison IOM showing a "fluffy" unstructured material (1) and submicron-sized spherical carbonaceous particles (2). Supporting grid (3). Courtesy of Laurence Garvie, Arizona State University. b) Hypothetical structure of the IOM [after 12]. 
the prebiotic pool of the early Earth. However, upon treatment with water in conditions of elevated temperature and pressure not dissimilar from those found in terrestrial hydrothermal vents, this kerogen-like material releases water-soluble organic molecules including long chain dicarboxylic acids up to seventeen-carbon long [13]. We may then as yet speculate that, were these compounds freed on the early Earth, the long-chain dicarboxylic acids could have been helpful in molecular evolution by aiding in the formation of vesicles for the encapsulation and efficient concentration of early prebiotic compounds or catalysts.

However, it has been the discovery and subsequent analytical characterization of Murchison soluble organic compounds that were immediately framed in the context of the origin of life and have had a profound significance for Exobiology, as they suddenly shed a unique light on the question of whether abiotic organic compounds could be linked to the development of planetary life.

Murchison soluble organics comprise a varied and complex suite of compounds that spans the range from large species such as polycyclic aromatic hydrocarbons or long alkyl acids and di-acids up to the limit of their solubility to small molecules like methane, formic acid or glycine. These compounds are listed in Table 1, however, their accurate representation is limited by the abundance, structural diversity and almost complete isomerism of the various of molecular types detected. As a general characteristic, they show exponential decline of abundance within the homologous series and a predominance of branched chain isomers. Murchison has a diverse and complex molecular composition that stands in clear contrast with the selective specificity of life's molecules and appears to be the product of purely physico-chemical processes, as well as a mark of a-biotic chemistry.

Nevertheless, within this complexity, several of the meteoritic compounds have identical counterparts in biochemistry, e.g., eight protein amino acids ( $g l y$, ala, val, leu, ile, pro, asp, $g l u$ ), pyridine carboxylic acids (e.g., nicotinic acid), sugar alcohols and several others that can be commonly found in extant organisms. Most intriguingly also, a subgroup of Murchison amino acids, the 2-methyl-2-amino acids, display small but significant L-enantiomeric excesses (ee) that are detected throughout their homologous series and range 1-18 $\%[14]$. This is the same configuration of all (except one, gly is not chiral) the amino acids that make up terrestrial protein and, given the pervasive chiral homogeneity of biomolecules, it would seem reasonable to suspect that meteoritic ee could be the product of terrestrial contamination. However, the $\alpha$ branched amino acids are not components of proteins or very common in the biosphere; moreover, their chiral asymmetry in meteorites was established unambiguously by ${ }^{13} \mathrm{C}[15]$ and hydrogen [16] isotopic measurements, in fact, by this proof of their indigeneity, these non-racemic amino acids stand as the only natural sample of molecular asymmetry measured so far outside the biosphere.

Overall the molecular analyses of the Murchison meteorite allow the conclusion that abiotic chemical processes are
Table 1. Soluble organic compounds identified in the Murchison meteorite

\begin{tabular}{lcc}
\hline Compound Class & $\mathrm{n}^{1}$ & $\mathrm{ppm}^{2}$ \\
\hline Carboxylic acids & 31 & 300 \\
Amino acids & 74 & 60 \\
Hydroxy acids & 15 & 7 \\
Ketoacids & 5 & $\mathrm{nd}^{3}$ \\
Dicarboxylic acids & 17 & 30 \\
Sugar alcohols \& acids & 19 & 30 \\
Aldehydes \& Ketones & 18 & 20 \\
Amines \& Amides & 20 & 13 \\
Pyridine carboxylic acids & 7 & 7 \\
Purines \& Pyrimidines & 5 & 1.5 \\
Hydrocarbons: & & \\
Aliphatic & 140 & 35 \\
Aromatic & 25 & 30 \\
Polar & 10 & 120 \\
\hline
\end{tabular}

${ }^{1}$ Number of compounds identified (see text), ${ }^{2}$ parts per million, ${ }^{3}$ not determined.

capable of form diverse organic materials that can reach considerable complexity and may comprise compounds similar or identical to biomolecules. The molecular asymmetry of some meteoritic amino acids also gives evidence that the seemingly random abiotic syntheses that produced meteorite organics could include a degree of chiral selection, a molecular trait intimately associated with life.

\section{The long cosmic history of meteorite organics}

Undoubtedly, the understanding of the abiotic locales and physico-chemical processes that produced meteorites' organic compounds may bear profound implications for the study of the origin of life because it could lead to discover not only how molecules similar to biomolecules were formed abiotically but also how common or rare their distribution may be. Molecular analyses have not been sufficient for these insights, in fact, questions about the formative processes for meteoritic compounds were widely discussed during the 70 s and 80s [8] but all fell short of accounting for their diverse traits. A leap in understanding their formation came, at least in general terms, with the compound specific analysis of various meteoritic compounds' isotopic composition. This is because, as we know, different synthetic pathways in forming a molecule lead to different extent of mass-dependent isotopic fractionation of that molecule's component elements and, as a result, isotope distribution may represent the best tracer of its synthetic history.

For meteorite organics, the most informative studies were those of their $\mathrm{D} / \mathrm{H}$ composition, due to the fact that fraction- 
ation takes the form $\exp (-\Delta \mathrm{E} / \mathrm{T})$, i.e., is directly proportional to the difference in mass between isotopes and inversely proportional to the $\mathrm{T}$. This ultimately means that isotopic fractionation would be largest for $\mathrm{D} / \mathrm{H}$ at very low $\mathrm{T}$ and a demonstration of the theory was found in the D-enrichment of the molecules observed in the dense clouds of the interstellar medium (ISM). These are the vast expanses of gas and dust found in the space between the stars, held loosely together by gravity. The ISM is made of the material ejected by several generations of dead stars during their life span as well as upon their often-violent deaths and it is from this material that new stars are formed. Temperature and pressures in these regions can be extremely low $\left(5-10 \mathrm{~K}\right.$ and $10^{4} \mathrm{nH} \mathrm{cm}^{-3}$, respectively) and seemingly forbidding, yet, organic molecules are observed to form, both in the gas phase as well as on icy grain, and to have tremendous enrichments of deuterium (for an ISM DHO/ $\mathrm{H}_{2} \mathrm{O}$ 0.01 while the average terrestrial $\mathrm{D} / \mathrm{H}$ ratio is $1.510^{-4}$ ) [17].

Based on these data, the findings that Murchison organic compounds were all enriched, to various degrees, in the heavy isotopes of $\mathrm{H}, \mathrm{C}$ and $\mathrm{N}$ [8], linked them to chemical processes in very cold cosmic locals such as those of the ISM. Broadly stated, the current hypothesis proposes that the compounds we detect in the Murchison-type of meteorite, or their direct precursors, were synthesized in the cold molecular clouds of the ISM and further reacted through the subsequent stages of pre-stellar, nebular and asteroidal processes, which must have been mild enough for them to preserve the "cold" signature of those distant regimes.

\section{The Antarctica finds and their novel composition}

Overall, CC meteorites do not seem to differ much from terrestrial rocks, a similarity that has not helped their collection or preservation because, if not seen to fall and promptly collected, they easily disappear in the environment. The Murchison meteorite was exceptional in this respect, by falling at the time of interest for extraterrestrial materials, and its subsequent analyses represent the most comprehensive study of any extraterrestrial organic material to date. As a result of this focus, the Murchison's composition has for long been considered representative not only of meteorites of the same type but often also of the capabilities of abiotic organic syntheses in general [18]. Given our yet tentative knowledge of cosmochemical environments, it is not surprising that the latter assumption turned out to be premature and a new group of pristine meteorites collected in the ice fields of Antarctica, the Renazzo-family of chondrites or $\mathrm{CR}$, have been offering a novel and fascinating view of different synthetic outcomes from a-biotic chemical evolution as well as of their prebiotic relevance.

As for Murchison, the formative origin of the CR organic suite was traced back to presolar environments, in fact, the $+7200 \delta \mathrm{D}$ value determined for a CR $\alpha$-amino isobutyric acid is the highest ever measured directly for an extraterrestrial molecule and falls within the values determined spectroscopically for interstellar molecules [9]. Similarities between the two types of meteorites, however, end here and Figure 2 summarizes the uniqueness of CR meteorites' organic suite in comparison to Murchison's. While displaying diverse molecular species not unlike those of other CC, CRs appear exceptional for having a definite preponderance of water-soluble compounds (over $90 \%$ overall); novel are also the relative and absolute distributions of these compounds: ammonia is the largest single molecular component of CR2 extracts, as in Murchison, but it is larger than in Murchison by over an order of magnitude and N-containing amino acids and amines are not only substantially more abundant but also more so than O-containing mono-, di-, and hydroxy-carboxylic acids. The molecular distributions within several of the CR2 classes of compounds are also so far unique, for example, the lower molecular weight molecules of a homologous series, such as glycine, $\alpha$-amino isobutyric acid or glycolic acid, are dominant in the extracts and represent the bulk of the total amounts; in Murchison, by contrast, overall soluble compound abundances decrease linearly with increasing carbon chain length. In addition, both CR2 meteorites analyzed contain compounds found in lower abundance or absent in CM2 meteorites, such as aldehydes and ketones, hydroxy-amino acids (the protein amino acids serine, threonine and tyrosine never identified in meteorites before) as well as an abundant suite of tertiary amines.

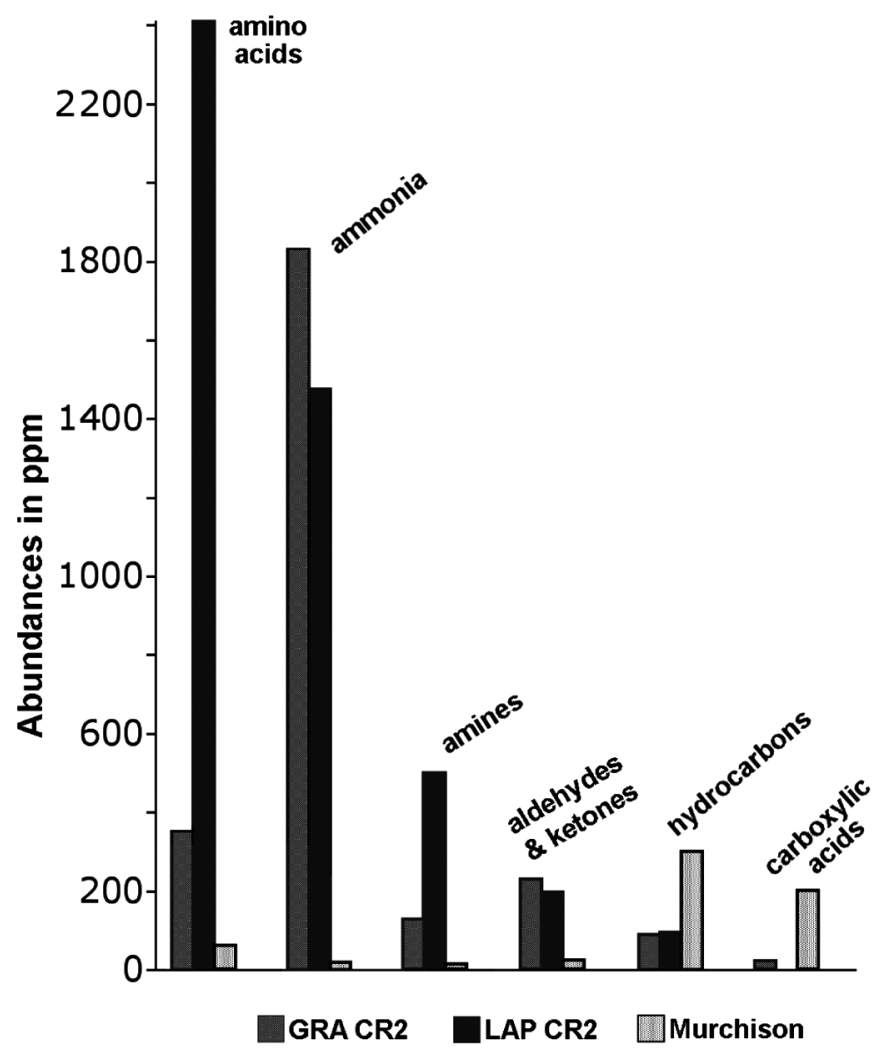

Fig. 2. Comparative plot of major soluble organic compound abundances in the Murchison and two CR2 meteorites (GRA 95229 and LAP 02342). 
Another puzzling difference between CR2 and Murchison-type meteorites regards their molecular asymmetry. In CR2s, the same 2-methyl amino acids amino acids found non racemic in Murchison display ee to less extent or not at all, while the study of the meteorites' leucine (leu) and alloisoleucine (allo) diastereomer amino acids allowed the inference of an original asymmetry of their precursor aldehydes [9]. This took a somewhat indirect reasoning and was explained as follows.

A possible reaction for the formation of amino acids in meteorites is the addition of $\mathrm{HCN}$ to ketones and aldehydes in the presence of water and ammonia (Fig.3, a). Although producing an asymmetric carbon in most cases (and therefore a chiral molecule), this type of synthesis is non-stereospecific because the HCN addition to the imine bond would be random and give equal amounts of D-, and L-enantiomers. In the case of the isoleucine diastereomers, however, the precursor aldehyde (2-methyl butyraldehyde) would have been chiral and, were an ee present in the aldehyde, e.g., of the (S) configuration, those amino acids that carried the S-portion of the molecule through their synthesis will be more abundant than their respective enantiomers. In the above example this would be the (RS) allo and (SS) ile compounds or, in the formalism used for amino acids, D-allo and L-ile (Fig.3, b). Such was the distribution found in the CR2 extracts (Fig.3, c) and on this basis, as well as the further confirmation obtained by isotopic analyses of the individual enantiomers [9], the findings were interpreted to signify that their precursor aldehyde carried an original S-asymmetry to the meteorite's asteroidal body.

When compared to Murchison composition and molecular distributions, the data obtained for CR2 meteorites offer a dramatic new outlook of the synthetic capabilities of cosmic environments and of the possible prebiotic relevance of their organic materials. Murchison's organic composition is unequivocally heterogeneous, the likely result of random formative processes and suggestive of a fundamental distinction between a-biotic and biological chemical processes in their paths to molecular complexity [18]. As a result, these studies left many questions unanswered about Murchison organics' potential for further evolution. Mainly, by what means, selective factors or even contingencies could a collection of so many compounds (over one thousand) ever reach the molecular specificity of biochemistry.

On the other hand, the yet limited analyses of CR2 organic materials already allow important conclusions, i.e., that a still unknown combinations of cosmochemical contingencies lead CR2 precursor environments to a selection of molecular species that are over abundantly water-soluble, $\mathrm{N}$-containing and of low molecular weight. This account of cosmochemical evolution's unexpected capabilities and their clear prebiotic appeal seem also to allow rephrasing the basic exobiological question of whether extraterrestrial organic compounds contributed to molecular evolution on the early Earth and ask how this evolution might have proceeded upon delivery of organic materials with CR or mixed CR and Murchison type composition.

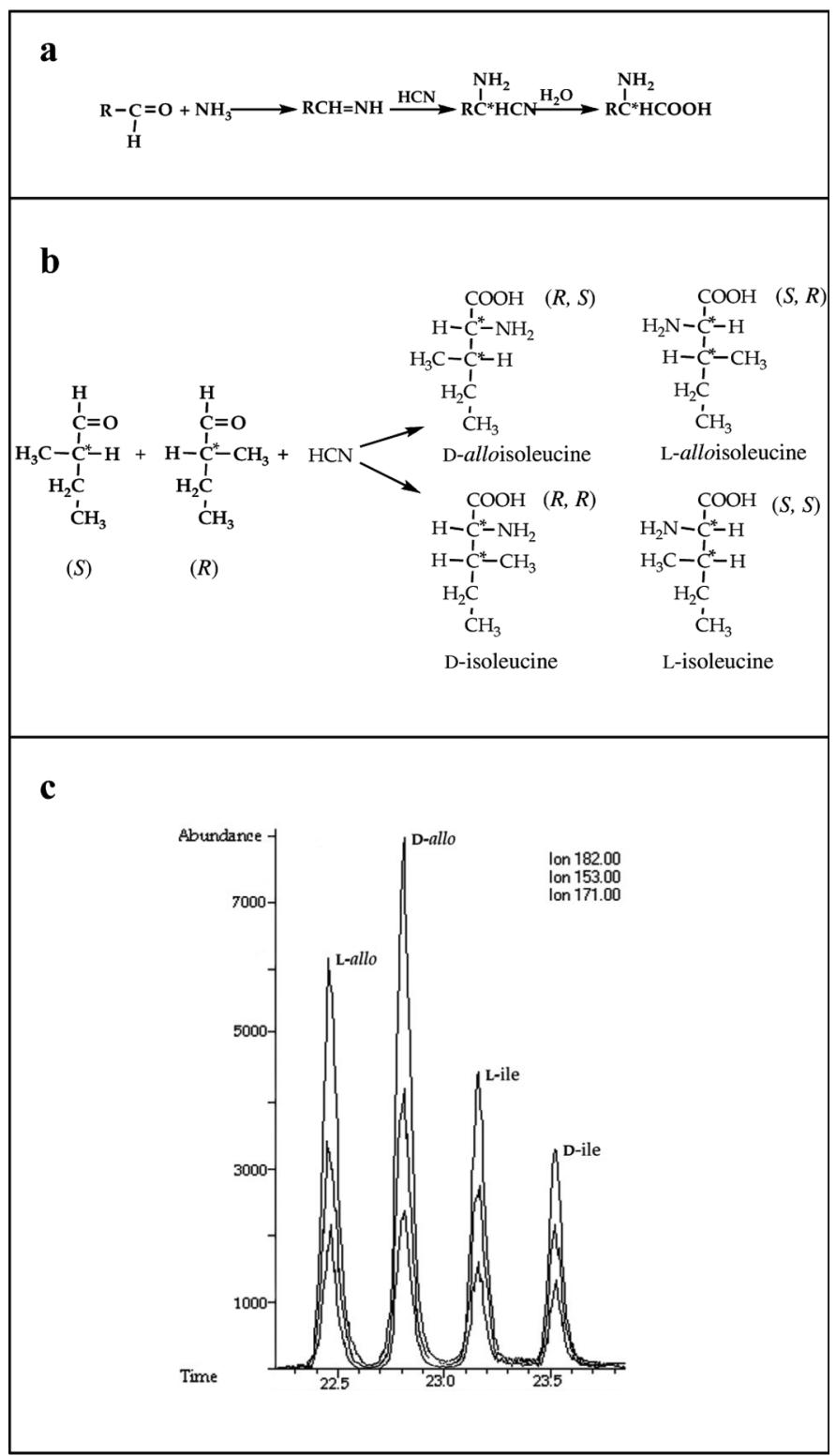

Fig. 3. a) General scheme of the cyanohydrin syntheses of amino acids from aldehydes; b) the synthesis of isoleucine and alloisoleucine from 2-methylbutyraldehyde; c) the chiral distribution of leucine diastereomers in the GRA95229 CR meteorite (by GC-MS on a Chrompack SP column).

\section{Exogenous delivery and the origin of life}

Although admitting to the multiple uncertainties about the origin(s) of life, the exobiological question is a reasonable one. Compounds we call biomolecules on the Earth are formed also abiotically and, it appears, in multiple cosmic environments. Through meteorites, these molecules have continuously showered the Earth and most intensively during the earlier Earth history [19]. Furthermore, if we concur with Eschenmoser that the origin of life cannot be discovered but only re-invented [20], in devising models towards that goal, we may consider paradigms whereby the organic molecules abundantly deliv- 


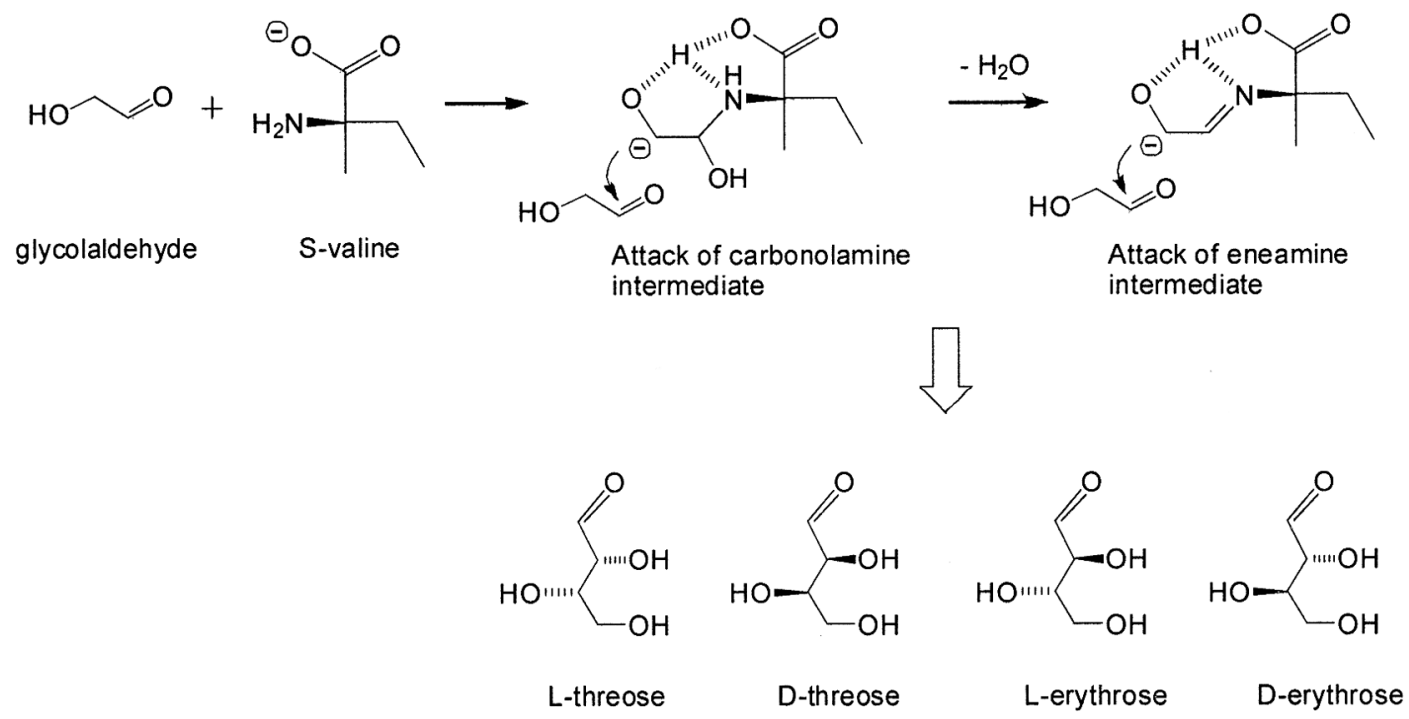

Scheme 1

ered by meteorites became enablers of molecular evolution. Between them, it is easy to single out meteoritic amino acids for prebiotic significance because these di-functional compounds, which in extant life are the basis of the structure and function of proteins as well biochemical catalysts, are over abundantly present in simple form in some of the meteorites analyzed as well as found non-racemic in other meteorites.

Following this rationale, the ability of amino acids to behave as asymmetric catalyst was tested through a series of prebiotically realistic experiments that modeled sugar synthesis from simple aldehyde reactants in water solutions. The prebiotic scenario assumed in these studies was: that the exclusive one-handedness of protein and nucleotide constituents (L-amino acids and D-sugars), which is now essential to extant biopolymers' structure and function, was also important to the emergence of life; that, because abiotic syntheses of chiral molecules would produce only racemic mixtures if unaided by catalysts, the development of chiral homogeneity might have been an evolutionary process that involved catalysis and that the unique chiral asymmetry of meteoritic amino acids might have transferred their asymmetry to other molecules essential to life.

It was first shown [21] that non-racemic isovaline (2-ethylglycine), an amino having up to near 20\% L-ee in Murchisontype meteorites, could affect catalytically the aldose condensation of glycolaldehyde producing tetrose sugars with significant ee. The asymmetric effect differed for the two 4C sugars, with L-isovaline producing ee for D-threose and L-erythrose, by the possible pathway shown in Scheme 1. Although diminishing with decreasing $e e$ of the catalyst, this effect persisted at levels of isovaline ee $(15 \%)$ seen in meteorites.

It was then demonstrated [22] that, using the same waterbased prebiotic model of sugar synthesis involving glycolaldehyde self-condensation, also homochiral L-dipeptide catalysts lead to the stereospecific syntheses of tetroses. In this case, the asymmetric effect is far larger and involves mainly erythrose that, with L-val-L-val catalyst, may reach a D-enantiomeric excess of over $80 \%$. Based on results obtained with various peptides, it was proposed that the possible catalytic reactionintermediate consisted of an imidazolidinone ring formed between the two nitrogen atoms of the peptide catalyst and the $\mathrm{C} 1$ of one-glycolaldehyde molecule (as shown in Scheme 2 for gly-L-val and gly-D-val catalysts).

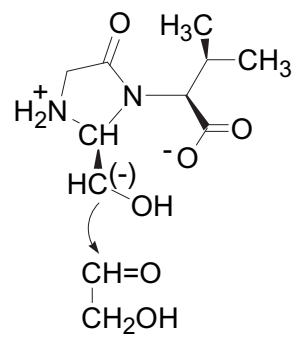

glycolaldehyde gly-L-val adduct up

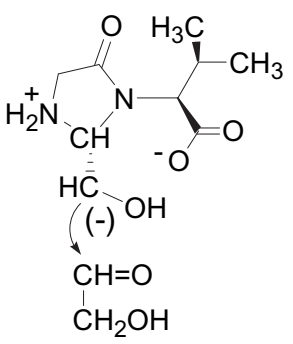

glycolaldehyde gly-L-val adduct down<smiles>COC(=O)[C@@H](C(C)C)N1C(=O)C[NH2+][C@H]1C</smiles>

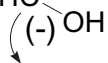<smiles>[CH]=O</smiles>$$
\mathrm{CH}_{2} \mathrm{OH}
$$

glycolaldehyde gly-D-val adduct up
Scheme 2

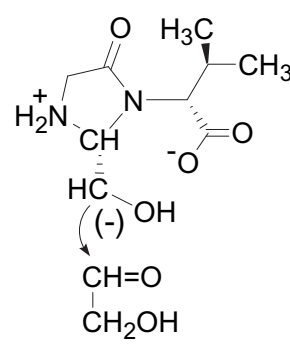

glycolaldehyde gly-D-val adduct down 


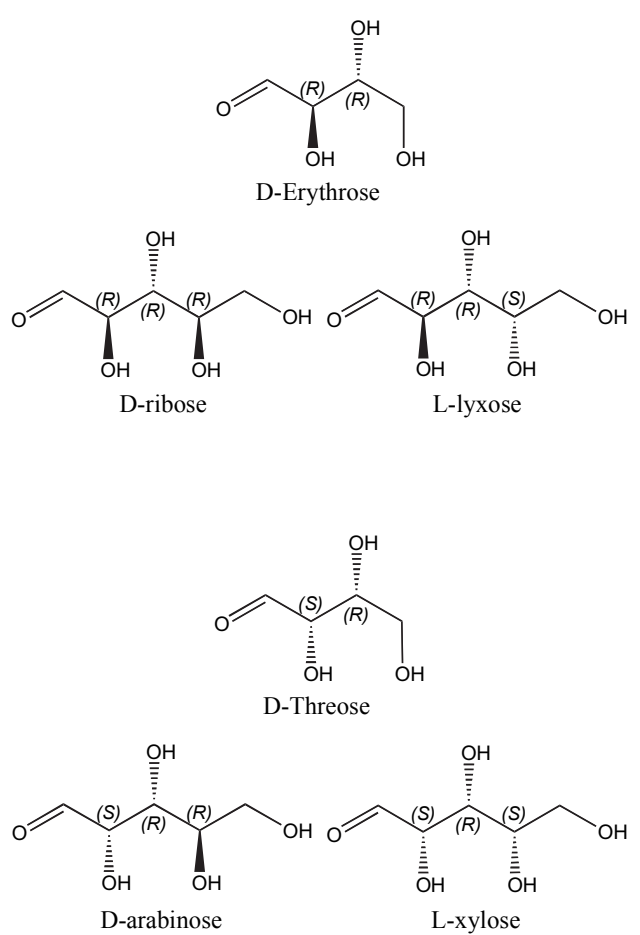

Scheme 3

Finally, it was shown recently [23] that the aldol condensation of glycolaldehyde and DL glyceraldehydes in the presence of homochiral or even scalemic dipeptide catalysts could produce pentose sugars whose configuration is affected by the chirality of the catalyst. All four 5-C sugars, lyxose, arabinose, ribose and xylose, were formed with varying ee and, of these, ribose was the only pentose sugar to have a significant ee $(\leq 44 \%)$ of configuration opposite to that of the catalyst (i.e., D-ribose ee with LL di-valine catalyst); lyxose and arabinose showed ee of the same sign instead, while the ee of xylose were minor if any. These syntheses were again explained via the formation of an imidazolidinone ring intermediate on the basis of similarities observed in the stereochemical configurations of the first two chiral carbons of tetroses and pentoses. As Scheme 3 shows, the comparison seem to relate the Dribose and L-lyxose, favored in the LL di-valine syntheses, to D-erythrose that was also shown to be favored by the same peptide in glycolaldehyde's self-condensation [20]. The LL divaline-unfavored L-ribose and D-lyxose are related to the unfavored L-erythrose as well, while arabinose and xylose, whose chiral distributions were not much affected by the catalytic syntheses, are likewise related to threose, also less affected in the catalyzed glycolaldehyde condensation.

To conclude, while we cannot yet answer the question of whether the diverse and complex chemistry observed in cosmic environments ever established a continuity into molecular evolution and life, on the Earth or elsewhere, the study of carbonaceous meteorites is very important to our quest to understand the emergence of life in that they provide to analysis an unparalleled record of prebiotic chemistry in the early Solar
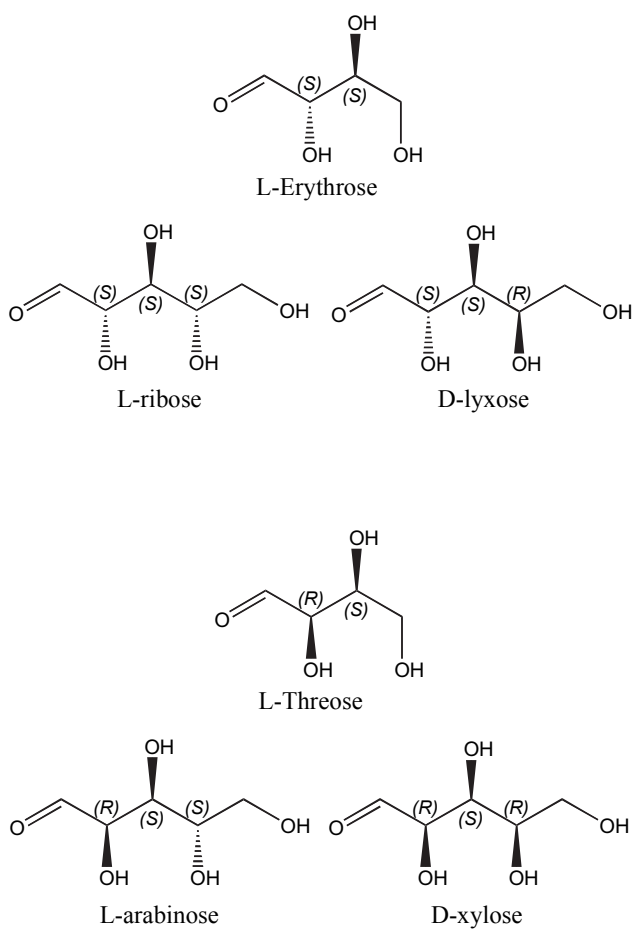

System and thus allow prebiotically realistic model experiments as well as the envisioning of evolutionary pathways to biogenesis.

\section{Acknowledgements}

I am grateful to Professor Eusebio Juaristi, Guillermo DelgadoLamas and Antonio Lazcano for their invitation and the opportunity to visit the Colegio Nacional. I also thank an anonymous referee for a very helpful review. These studies were supported through the years by grants from the Exobiology, Cosmochemistry and Origins of the Solar System programs of the US National Aeronautic and Space Administration.

\section{References}

1. In a letter to Joseph Hooker (1871), Darwin famously wrote: "It is often said that all the conditions for the first production of a living organism are now present, which could ever have been present. But if (and oh! what a big if!) we could conceive in some warm little pond, with all sorts of ammonia and phosphoric salts, light, heat, electricity, \&c., present, that a proteine compound was chemically formed ready to undergo still more complex changes, at the present day such matter would be instantly absorbed, which would not have been the case before living creatures were found".

2. Irvine, W. Origins Life Evol. Biosph. 1997, 28, 365-383.

3. Morbidelli, A. et al., Meteorit. Planet. Sci. 2000, 35, 1309.

4. Levison, H. F.; Bottke, W. F.; Gounelle, M.; Morbidelli, A.; Nesvorn, D.; Tsiganis, K. Nature 2006, 460, 364-366.

5. Hsieh, H. H.; Jewitt, D. Science 2006, 312, 561-563. 
6. Mullie, F.; Reisse, J. Top. Curr. Chem. 1987, 139, 83-117.

7. Kvenvolden, K.; Lawless, J.; Pering, K.; Peterson, E.; Flores, J.; Ponnamperuma, C.; Kaplan, J. R.; Moore, C. Nature 1970, 228 , 923-926.

8. Pizzarello, S.; Cooper, G. W.; Flynn, G. J. "Meteorites and the Early Solar System II", 2006, ed. D. S. Lauretta and H. Y. McSween Jr, University of Arizona Press, Tucson, AZ, 625-651.

9. Pizzarello, S.; Huang, Y.; Alexandre, M. D. R. P. Natl. Acad. Sci. USA 2008, 105, 7300-7304.

10. Pizzarello, S.; Holmes W. Geochim. Cosmochim. Acta 2009, 73, 2150-2162.

11. Garvie Garvie, L. A. J.; Buseck P. R. Earth Planet. Sci. Lett. 2004, 224, 431-439.

12. Hayatsu, R.; Matsuoka, S.; Scott, R. G.; Studier, M. H.; Anders E. Geochim. Cosmochim. Acta, 1977, 41, 1325-1339.

13. Yabuta, H.; Williams, L. B.; Cody, G. D.; Alexander, C. M. O’D.; Pizzarello S. Meteorit. Planet. Sci. 2007, 42, 37-48.
14. Cronin, J. R.; Pizzarello, S. Science 1997, 275, 951-955.

15. Pizzarello, S.; Zolensky, M.; Turk, K. A. Geochim. Cosmochim. Acta 2003, 67,1589-1595.

16. Pizzarello, S.; Huang, Y. Geochim. Cosmochim. Acta 2005, 69, 599-605.

17. Roueff, E.; Gerin, M. Space Sci. Rev. 2003, 106, 61-72.

18. Pizzarello, S. Chem. Biodivers. 2007, 4, 680-693.

19. Chyba, C. F.; Sagan, C. Nature 1992, 355, 125-132.

20. Eschenmoser, A. The Scripps Research Institute News and Views, 2008, 8(13), http://www.scripps.edu/newsandviews/e_20080421/

21. Pizzarello, S.; Weber, A. L. Science 2004, 303, 1151.

22. Weber, A. L.; Pizzarello, S. P. Natl. Acad. Sci. USA 2006, 103, 12713-12717.

23. Pizzarello, S.; Weber, A. L. Origins Life Evol. Biosph. 2010, 40, 3-10. 